\title{
Influencia del cambio climático en la biología de Aedes aegypti (Diptera: Culicidae) mosquito transmisor de arbovirosis humanas
}

\author{
María A. López-Latorre ${ }^{1}$ y Marco Neira ${ }^{1} \square$ \\ ${ }^{1}$ Centro de Investigación para la Salud en América Latina (CISeAL), Escuela de Ciencias Biológicas, Pontificia \\ Universidad Católica del Ecuador, Av. 12 de Octubre 1076 y Roca, Quito, Ecuador. \\ $\nabla$ mvneira@puce.edu.ec
}

\section{Recibido 19-02-2016, Aceptado 04-04-2016}

\begin{abstract}
RESUMEN.- En la actualidad, las enfermedades transmitidas por el mosquito Aedes aegypti, incluyendo las fiebres del dengue, chikungunya y Zika, se encuentran entre los principales problemas de salud en países tropicales. Si se considera que la biología de esta especie de insecto está influenciada en gran medida por las condiciones ambientales, podemos afirmar que los cambios previstos en el clima futuro del planeta tendrán un impacto considerable en el panorama epidemiológico de las enfermedades transmitidas por A. aegypti.

En el presente trabajo revisamos el estado del conocimiento actual sobre la influencia que factores ambientales pueden tener en la biología y fisiología de A. aegypti. Adicionalmente, discutimos cómo los cambios climáticos previstos para el futuro pueden afectar la capacidad vectorial de esta importante especie, con especial énfasis en el Ecuador y sus países vecinos.
\end{abstract}

PALABRAS CLAVES: Cambio climático, Aedes aegypti, mosquito, dengue, chikungunya, Zika

\begin{abstract}
The suite of diseases currently being transmitted by the mosquito Aedes aegypti, including dengue, chikungunya and Zika, are among the biggest threats to human health in tropical countries. Considering that the biology of this insect species is significantly influenced by environmental conditions, we can state that the any changes in the planet's future climate will have a considerable impact on the epidemiological landscape of all diseases vectored by $A$. aegypti. In this paper, we review our current knowledge regarding the influence that environmental factors can exert on the biology and physiology of A. aegypti. Furthermore, we discuss the ways in which future climate variations can influence vector capacity in this species, with special emphasis in Ecuador and its neighboring countries.
\end{abstract}

KEYWORDS: Climate change, Aedes aegypti, mosquito, dengue chikungunya, Zika

El cambio climático es un fenómeno definido por las variaciones significativas y duraderas de los patrones locales o globales del ambiente. Este proceso está estrechamente ligado con el calentamiento del sistema climático, proceso también conocido como calentamiento global (NASA 2015, IPCC 2013). En la actualidad, el cambio climático es considerado uno de los problemas ambientales más importantes y se prevé que este fenómeno se vuelva aún más relevante a nivel global en las próximas décadas.

El efecto que el cambio climático puede tener sobre la salud humana es complejo, y para entenderlo es necesario analizar los vínculos entre el clima actual, el clima futuro, y los principales problemas de salud en el mundo (Epstein 2005). El clima influye de forma importante en las enfermedades infecciosas y sus diferentes modos de transmisión: vectorial, agua, alimentos, suelos, mar y aire, siendo el efecto más pronunciado el observado en las patologías transmitidas por vectores (Morin et al. 2013).

Uno de los grandes desafíos científicos actuales es comprender la manera en la cual el cambio climático global afectará al paisaje epidemiológico futuro. En lo referente a las enfermedades arbovirales, aquellas transmitidas por el mosquito Aedes aegypti (incluyendo a la fiebre del dengue, chikungunya y Zika) se cuentan entre las de mayor relevancia para la salud pública. Debido al pronunciado efecto que los cambios ambientales pueden ejercer sobre la biología de $A$. aegypti, es muy probable que la epidemiología de estas arbovirosis se vean profundamente influenciadas por el cambio climático futuro.

El propósito del presente artículo es el revisar el conocimiento disponible sobre los aspectos de la biología de 
A. aegypti que son directamente influenciados por factores ambientales, y ponerlos en el contexto del cambio climático global. Con esto, esperamos contribuir al entendimiento de cómo los potenciales cambios futuros en patrones climáticos pueden afectar a la epidemiología de enfermedades transmitidas por A. aegypti.

Aedes aegypti y las arbovirosis humanas.- A. aegypti (Diptera: Culicidae) es una especie de mosquito originaria del África, (Figura 1). Debido a su alta capacidad para adaptarse a sobrevivir en asentamientos humanos, A. aegypti se ha extendido por todo el planeta, generalmente a lo largo de rutas comerciales y turísticas (Cook \& Zumla 2009, Marquardt 2005, WHO 2015).

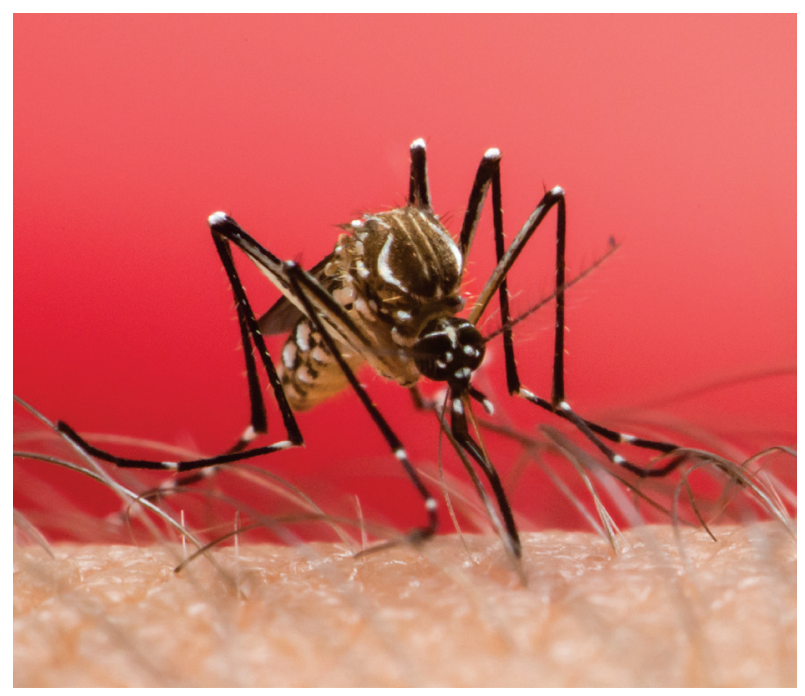

Figura 1. Aedes aegypti (Diptera: Culicidae). Al alimentarse de sangre, las hembras de esta especie de mosquito son responsables por la transmisión de varias enfermedades virales de gran importancia para la salud pública a nivel mundial. Fotografía cortesía de Esteban Baus / CISeAL.

Debido a su capacidad para la transmisión de varios arbovirus que afectan la salud humana, esta especie se ha tornado en uno de los principales vectores de enfermedad alrededor del planeta.

El término arbovirus proviene de la abreviación inglesa del término "arthropod-borne-virus", que significa "virus transmitido por artrópodos". Los arbovirus sobreviven en la naturaleza mediante la transmisión desde hospederos vertebrados infectados a hospederos susceptibles, a través de la picadura de artrópodos hematófagos.

Algunos arbovirus son transmitidos a los seres humanos por mosquitos (insectos pertenecientes al orden Diptera, familia Culicidae). A. aegypti es el principal vector de cuatro arbovirosis humanas: fiebre amarilla, fiebre del dengue, fiebre chikungunya y fiebre Zika (Cook \& Zumla, 2009). Estas patologías arbovirales se encuentran entre las enfermedades infecciosas emergentes más importantes y constituyen problemas de salud pública a nivel global (Uyar 2013, Gubler 2001).

La fiebre amarilla es una enfermedad generalmente transmitida en las áreas tropicales y sub-tropicales de África y América. El agente etiológico de esta patología es el virus de la fiebre amarilla, un arbovirus perteneciente a la familia Flaviviridae que presenta 7 genotipos diferentes, de los cuales 5 circulan en África y 2 en América (Cook $\&$ Zumla, 2009). Esta enfermedad fue controlada hace varias decadas gracias a la inmunización de la población con una vacuna altamente eficaz, segura y económica (Gubler 2001).

Una de las arbovirosis humanas más importantes es la fiebre del dengue, que se distribuye en regiones tropicales y subtropicales de todo el planeta (Bhatt et al. 2013). La enfermedad tiene como agente etiológico al virus del dengue, que pertenece a la familia Flaviviridae. Existen cuatro serotipos distintos del virus del dengue (DENV1, DENV-2, DENV-3 y DENV-4), los cuales presentan diferencias en la composición de su genoma y sus antígenos de superficie (Vaughn et al. 2000, Kuhn et al. 2002). La severidad de la enfermedad causada por la infección con el virus del dengue puede variar entre casos asintomáticos y casos fatales. A pesar que no se sabe a ciencia cierta cuáles son los factores que determinan la evolución de la enfermedad en un paciente, se cree que la infección secuencial con distintos serotipos del virus puede ser un factor desencadenante de la presentación más severa de la enfermedad, y puede llegar a tener consecuencias fatales (Vaughn et al. 2000).

La fiebre del dengue es la enfermedad transmitida por mosquitos que se ha propagado más rápidamente en el mundo (WHO/TDR 2009). La Organización Mundial de la Salud (WHO, por sus siglas en inglés) y el Programa Especial para la Investigación y Capacitación de Enfermedades Tropicales (TDR, por sus siglas en inglés), reportan que anualmente se producen aproximadamente 50 millones de infecciones por dengue, y alrededor de 2.5 mil millones de personas viven en países con dengue endémico (WHO/TDR 2009). En contraste, estudios recientes estiman que los datos de la WHO podrían subestimar la incidencia real del dengue, pues no toman en cuenta los casos asintomáticos. Bhatt y sus colaboradores (2013) han propuesto que el número de nuevas infecciones con este virus puede llegar a sobrepasar los 300 millones cada año.

En Ecuador, el Ministerio de Salud Pública (MSP) estima que el $70 \%$ de la superficie terrestre del país tiene condiciones ambientales adecuadas para la transmisión de la fiebre del dengue, lo cual pone en riesgo a aproximadamente 8’220 000 de ecuatorianos (MSP 2013). 
Según el MSP, el promedio anual de casos de dengue entre los años 2011 y 2014 es de 13235 (MSP 2015). Durante el año 2015, el número total de casos de dengue reportados en territorio ecuatoriano fue de 42505 (Figura 2), cifra que representa más del doble de casos que en cualquiera de los cuatro años previos (MSP 2015). Sin embargo, es importante considerar que una porción (tal vez considerable) de estos casos podría corresponder a diagnósticos erróneos de casos de infección con el virus de chikungunya. La infección con chikungunya genera cuadros sintomáticos que pueden confundirse con el dengue y, debido a la reciente introducción del virus de chikungunya en el territorio nacional, es posible que los protocolos tradicionales de diagnóstico no hayan sido lo suficientemente sofisticados como para diferenciar entre estas enfermedades.

Por otro lado, el virus del chikungunya fue aislado por primera vez en Tanzania y recibe su nombre del idioma Makonde, en el cual la palabra "chikungunya" puede entenderse como "persona retorcida", en referencia a los fuertes dolores articulares que produce la enfermedad (Cook \& Zumla 2009, Gould \& Higgs, 2009). El agente causal de la enfermedad es un Alphavirus de la familia Togaviridae (Powers \& Logue, 2007). Esta patología tradicionalmente se encontraba en África, Asia y el subcontinente Indio; sin embargo, en las últimas décadas se han reportado brotes en nuevas regiones, con inclusión del continente Americano (OMS 2015, Powers \& Logue 2007). En el Ecuador, esta enfermedad se reportó por primera vez en el año 2014, con tan solo 27 casos identificados. Sin embargo este número se incrementó drásticamente durante el siguiente año, y llegó a un total de 33669 casos (MSP 2016a). Estas cifras resaltan el enorme potencial epidémico de esta enfermedad, el cual es característico de las enfermedades transmitidas por vectores.

Finalmente, el virus del Zika, perteneciente a la familia Flaviviridae, es el agente etiológico de la enfermedad conocida como fiebre Zika (Duffy 2009). Los brotes de Zika se han producido en zonas de África, el sudeste de Asia, las islas del Pacífico y, muy recientemente, las Américas (CDC 2016). El Ministerio de Salud del Ecuador en el año 2015 decretó una alerta epidemiológica para la fiebre del Zika en base de los diferentes casos que se presentaron en Colombia (MSP 2016b). A pesar de las precauciones tomadas por las autoridades de salud del país, para inicios del 2016 ya se habían confirmado casos de Zika en territorio ecuatoriano (Figura 2), incluyendo casos de transmisión local.

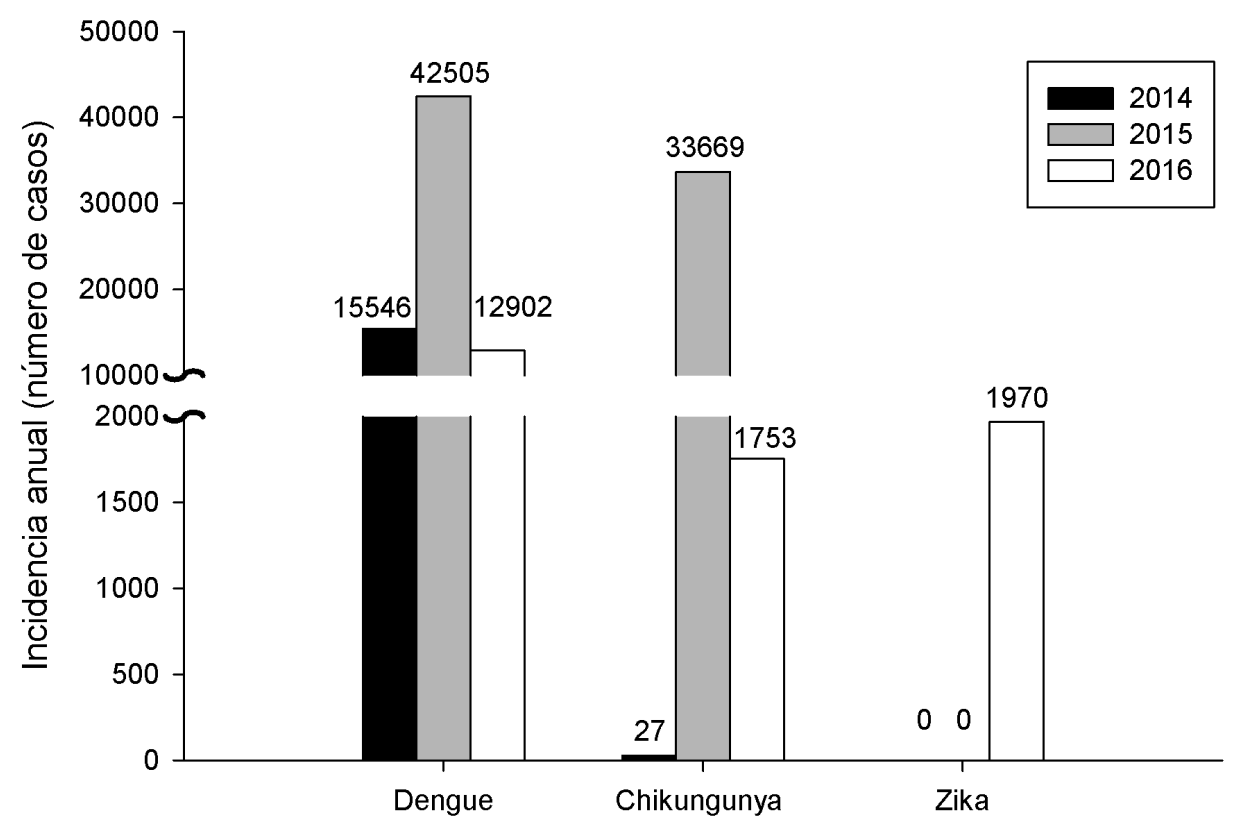

Figura 2. Situación de las arbovirosis transmitidas por A. aegypti en el Ecuador. Se muestra la incidencia de las fiebres del dengue, chikungunya y Zika durante los tres años más recientes. La fiebre Zika se detectó por primera vez en el Ecuador en el año 2016. Datos de Zika mostrados corresponden el número de casos confirmados reportados hasta la semana epidemiológica 33 del año 2016; los datos de la fiebre del dengue para el año 2016 corresponden el número de casos confirmados reportados hasta la semana epidemiológica 37 de ese año, y los datos de chikungunya para el año 2016 corresponden a casos confirmados hasta la semana epidemiológica 38 de este año. No se muestran datos sobre la fiebre amarilla debido a que no se han reportado casos de esta enfermedad en el Ecuador desde el año 2013. Los números mostrados sobre cada barra representan la incidencia anual correspondiente a cada enfermedad. Fuentes de datos: MSP 2015, 2016a, 2016c, 2016e; PAHO 2016. 
Hasta la semana epidemiológica 33 de ese año, se reportaron 1970 casos de Zika en el Ecuador (MSP 2016c, 2016d). A excepción de la fiebre amarilla, hasta el momento no existe una vacuna comercial para las otras tres principales enfermedades arbovirales transmitidas por A. aegypti, y tampoco existe un tratamiento específico; debido a esto, el único método de control de estas enfermedades es el evitar el contacto entre los seres humanos y el mosquito transmisor. Para esto, los métodos más efectivos incluyen la reducción de las poblaciones de A. aegypti y la aplicación de métodos que reduzcan el número de picaduras potencialmente infecciosas (tales como el uso de repelentes, la instalación de mallas en puertas y ventanas, y el uso de prendas de vestir apropiadas). Adicionalmente, toda mejora en la calidad de vida y los niveles de educación de la población en riesgo, generalmente conllevan a una reducción de la incidencia de estas enfermedades.

En la actualidad se utilizan dos mecanismos principales de control para poblaciones de A. aegypti: el uso de pesticidas químicos (ya sean adulticidas o larvicidas), y la reducción de los sitios de criaderos (Ranson et al. 2010). Desafortunadamente, ambos mecanismos tienen inconvenientes significativos. En primer lugar, la eficacia de los insecticidas comerciales es limitada debido a la aparición de poblaciones resistentes a los pesticidas (Ocampo et al. 2011) y a las dificultades logísticas del proceso requerido. En segundo lugar, la reducción de los sitios de reproducción es un proceso costoso y difícil de implementar, ya que depende de personal debidamente capacitado para la búsqueda y el tratamiento o eliminación de las áreas que presenten características ambientales idóneas para el desarrollo de criaderos de A. aegypti.

Las poblaciones de A. aegypti transmiten enfermedades arbovirales de forma exitosa cuando las condiciones ambientales son las adecuadas (Cook \& Zumla 2009). Los componentes ambientales son críticos para la supervivencia y reproducción de $A$. aegypti, además el clima influye de manera sustancial en diferentes aspectos fisiológicos (Bar-Zeev 1958, Tun-Lin 2000) y etiológicos (Barrera et al. 2011, Chowell et al. 2011) tanto de larvas como adultos y en la interacción del vector con los patógenos (Watts et al. 1986), lo que convierte a la transmisión de arbovirosis humanas en un proceso altamente sensitivo al ambiente (Morin et al. 2013). Por esta razón, es fundamental detallar la situación ambiental actual en las zonas en donde las arbovirosis constituyen un problema de salud grave. Además, es necesario comprender cómo estas variaciones ambientales pueden influir en las poblaciones de A. aegypti, y en su capacidad para la transmisión de enfermedades.
Cambio climático, variaciones observadas y proyecciones futuras.- Existen procesos naturales y antropogénicos que alteran el balance energético de la Tierra y son el motor físico del cambio climático (IPCC 2013). Dentro de las causas naturales del cambio climático se encuentran las variaciones en la energía solar absorbida, los aerosoles residuales de erupciones volcánicas, la circulación oceánica y los procesos biológicos, entre otros. (IPCC 2013).

En contraste, el cambio climático por influencia antropogénica hace referencia a todos los cambios ambientales asociados a actividades humanas. En primer lugar, podemos destacar el efecto combinado del incremento en la concentración atmosférica de varios gases asociados al efecto invernadero (es decir, gases que atrapan calor en la atmósfera terrestre, tales como el dióxido de carbono, metano y óxido nitroso) y, en segundo lugar, el uso no controlado de aerosoles (Santer et al. 1996) compuestos por clorofluorocarbonos y azufre que, además, afectan a la capa de ozono de la atmósfera (NASA 2015). Se estima que la contribución de los efectos antropogénicos al cambio climático es sustancialmente más alta que la de los efectos naturales (IPCC 2013).

En la actualidad, las concentraciones atmosféricas de gases de efecto invernadero se encuentran en niveles que no tienen precedentes en los últimos 800000 años (IPCC 2013). Desde 1750, las concentraciones de dióxido de carbono $\left(\mathrm{CO}_{2}\right)$, metano $\left(\mathrm{CH}_{4}\right)$ y óxido nitroso $\left(\mathrm{N}_{2} \mathrm{O}\right)$, han aumentado en $40 \%, 150 \%$ y $20 \%$, respectivamente (Santer et al. 1996). Entre los gases que influencian en el efecto invernadero, el $\mathrm{CO}_{2}$ es el más importante. La acumulación atmosférica de $\mathrm{CO}_{2}$ ha aumentado a un ritmo acelerado, y ha causado que aproximadamente la mitad de las emisiones antropogénicas de $\mathrm{CO}_{2}$ acumuladas entre los años 1750 y 2011 se han producido en los últimos 40 años (IPCC 2013).

Cambios en la estructura vertical de la temperatura atmosférica han sido propuestos como la huella digital de la acumulación de los gases de efecto invernadero que inducen el cambio climático (Santer et al. 1996). Los gases acumulados en la atmósfera terrestre elevan la temperatura del planeta. El incremento de la temperatura media anual de la Tierra se define como calentamiento global (Allison et al. 2009). Los datos globales, combinando el promedio de la temperatura de la superficie terrestre con la superficie marina, muestran una tendencia lineal de calentamiento promedio de $0.85^{\circ} \mathrm{C}$ en el período comprendido entre los años 1880 y 2012 (Figura 3A) (IPCC 2013). 


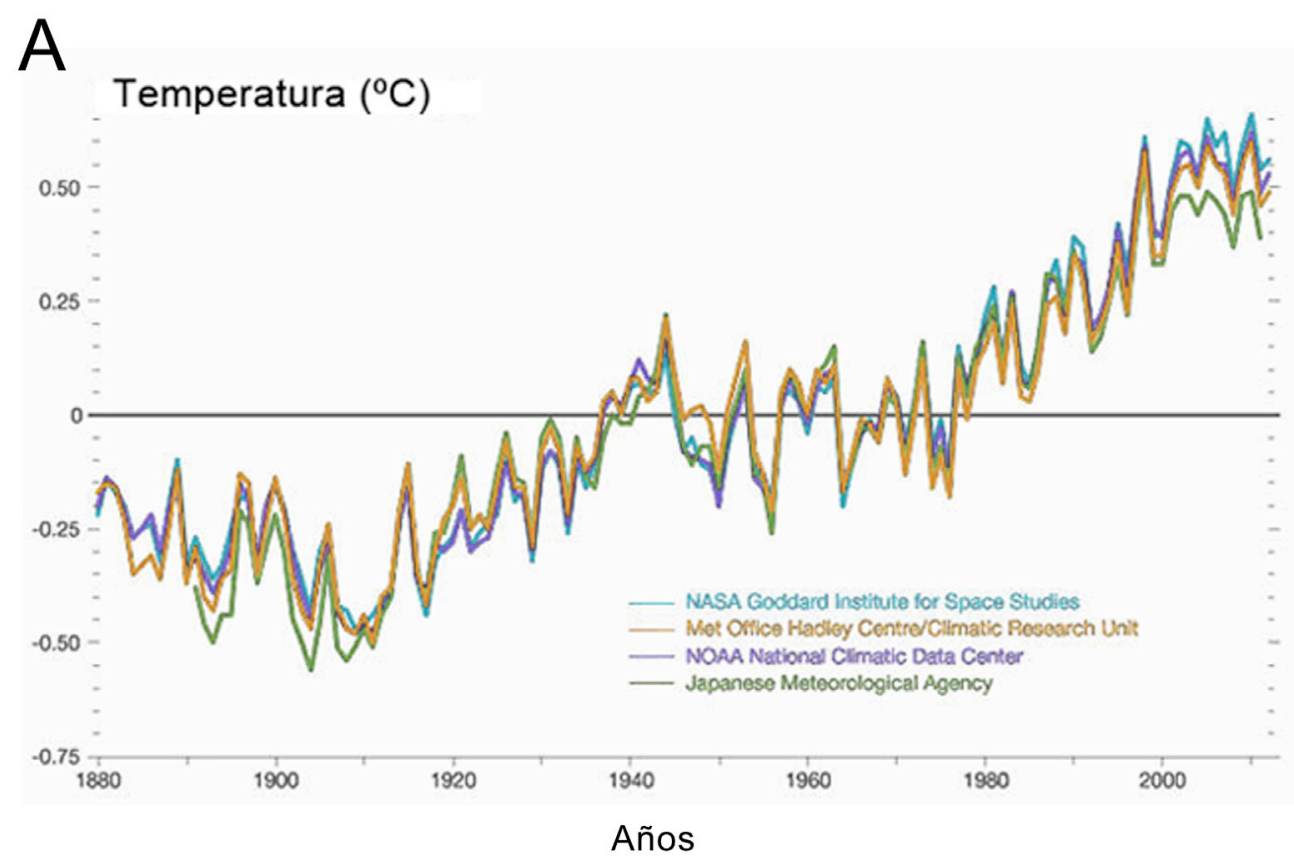

B

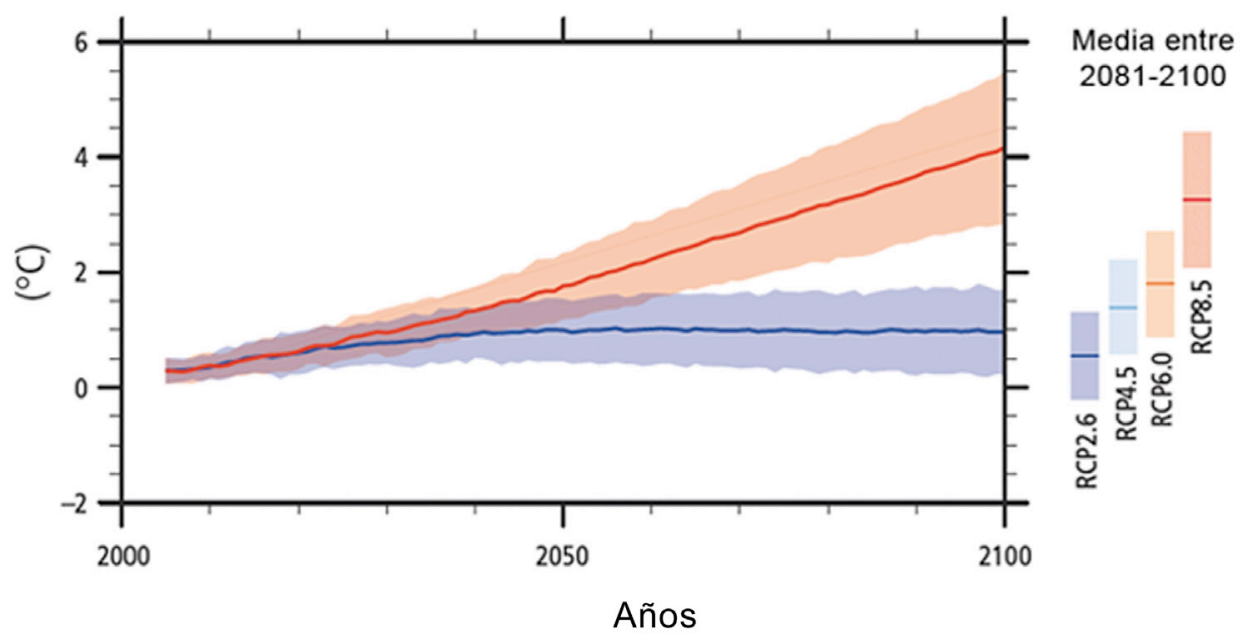

Figura 3. Evidencias de la influencia del cambio climático en la media de la temperatura del planeta. A) Variación de la temperatura promedio global de la superficie terrestre y oceánica combinadas desde el año 1880 hasta el año 2010. Cada una de las distintas curvas mostradas representa los datos generados por un grupo de investigación distinto (ver leyenda embebida en el gráfico para detalles). Como puede observarse, existe un alto nivel de consistencia entre los datos generados por diversos grupos independientes. B) Proyección del cambio en la temperatura global promedio para el período 2081- 2100, en relación al periodo comprendido entre 1986 y 2005. Se presentan diferentes escenarios de cambio, en función de distintos niveles de acumulación de gases de efecto invernadero en la atmósfera terrestre. Las potenciales concentraciones futuras de gases de efecto invernadero en la atmósfera terrestre se estiman utilizando modelos llamados "rutas de concentración representativa" (RCPs, por su siglas en inglés). La curva azul representa una proyección del cambio de temperatura en base a un RCP en el que la concentración de gases de efecto invernadero se mantiene en niveles mínimos (escenario denominado RCP2.6), mientras que la curva roja muestra la proyección del cambio de temperatura con un escenario en el que la concentración de gases de efecto invernadero es muy elevada (escenario denominado RCP8.5). Panel A cortesía de: NASA/JPL-Caltech. Panel B tomado de IPCC, 2013 (imagen de acceso libre disponible en: http://www.ipcc.ch/pdf/assessment-report/ ar5/syr/AR5_SYR_FINAL_SPM.pdf). 
Con el fin de obtener las proyecciones del cambio climático a largo plazo, se han desarrollado modelos climáticos predictivos y representaciones matemáticas que utilizan datos tales como la información descrita en los escenarios de acumulación de gases de efecto invernadero, las emisiones de contaminantes atmosféricos y los patrones de uso de la tierra. En comparación con 18501900 , se prevé que el promedio de la temperatura global de la superficie terrestre, para el final del siglo 21 (años 2081 a 2100) se incrementará de 1.5 a $2^{\circ} \mathrm{C}$ (Figura 3B) (IPCC 2013). Según la cantidad de gases de efecto invernadero acumulados a futuro en la atmósfera terrestre, la magnitud de este incremento podría alcanzar los $7^{\circ} \mathrm{C}$ (Figura 4A). (Allison et al. 2009).
Producto del aumento de la temperatura del planeta se han originado diversos cambios ambientales: el ciclo global del agua se ha afectado, el océano se ha calentado, las cantidades de nieve y el hielo en el planeta han disminuido y el nivel del mar se ha elevado. Las influencias antropogénicas han afectado el ciclo global del agua, probablemente desde 1960 (IPCC 2013). Se han observado aumentos en el contenido de humedad del aire en zonas templadas, y cambios a escala global en los patrones de precipitación sobre la tierra. Además, en las zonas del planeta donde la evaporación es mayor (trópicos y sub-trópicos) se ha visto una disminución de la humedad (Figura 4B) (IPCC 2013). En muchas regiones, los cambios en las precipitaciones o el derretimiento de la nieve y el hielo están alterando los sistemas hidrológicos, afectando a los recursos hídricos en cantidad y calidad (NASA 2015).

\section{A}

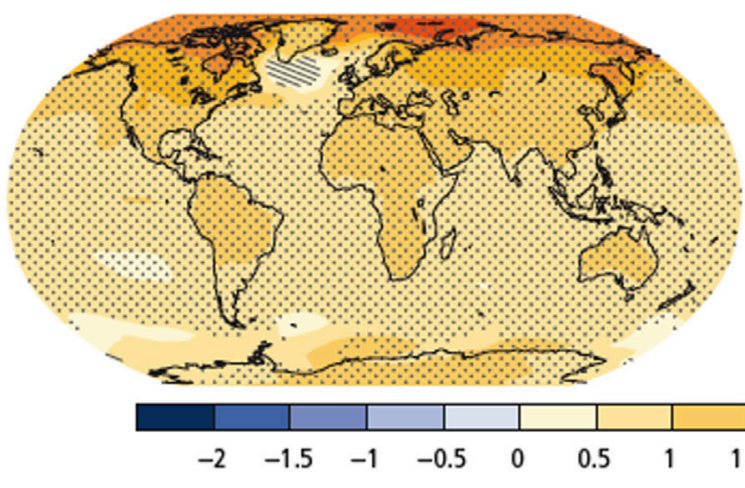

RCP2. 6

B

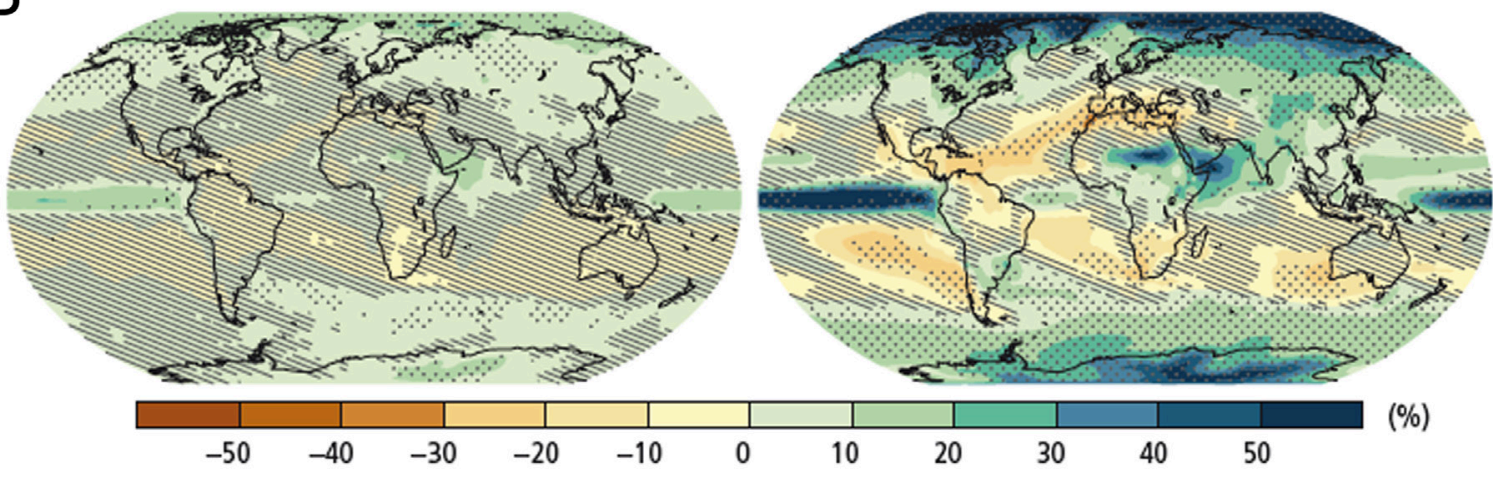

RCP8.5

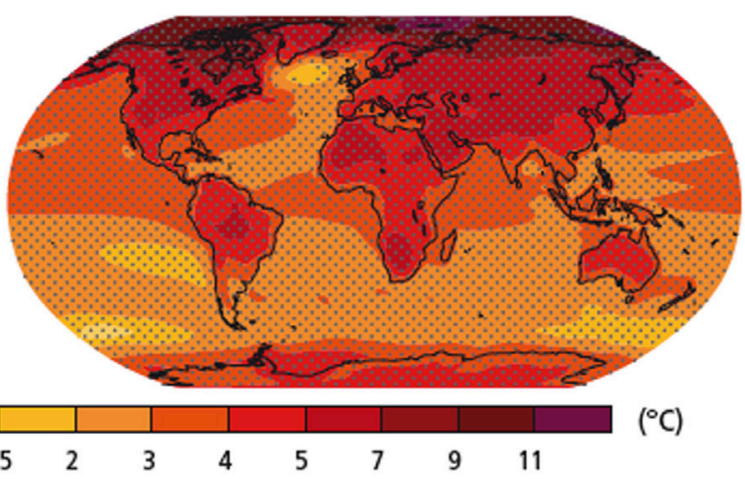

C)
B

Figura 4. Proyección de la influencia del cambio climático en la temperatura y precipitación del planeta. A) Proyección del cambio en la temperatura promedio de la superficie terrestre, comparando la media de la temperatura registrada entre los años 1986-2005 con la estimación la temperatura media entre los años 2081-2100. La estimación de la temperatura futura se realiza en combinación con modelos que predicen la concentración de gases de efecto invernadero en la atmósfera terrestre a largo plazo, conocidos como RCPs (ver leyenda de la figura 3 para una explicación más detallada sobre estos modelos). El panel izquierdo muestra cambio de temperatura con el modelo RCP2.6, que considera un escenario con niveles mínimos de concentración de gases de efecto invernadero, mientras que el panel derecho muestra la proyección del cambio de temperatura con un escenario en el que la concentración de gases es muy elevada (modelo RCP8.5). B) Proyección del cambio en los patrones de precipitación del planeta considerando dos modelos de diferentes escenarios de concentración de gases de efecto invernadero, RCP2.6 para el panel izquierdo, y RCP8.5 para el panel derecho. Paneles A y B tomados de IPCC, 2013 (imagen de acceso libre disponible en: http://www.ipcc.ch/pdf/ assessment-report/ar5/syr/AR5_SYR_FINAL_SPM.pdf). 
Además de los cambios en los patrones de precipitación y de humedad del planeta (Figura 4B), la absorción oceánica de $\mathrm{CO}_{2}$ ha dado lugar a la acidificación de los océanos y cuerpos de agua desde el comienzo de la era industrial (Gleick 1989, IPCC 2013). El pH del agua de la superficie del océano ha disminuido en 0.1 , lo que corresponde a un aumento del $26 \%$ en la acidez del agua (IPCC 2013).

En América Latina, se prevé que para el año 2100 la temperatura aumente entre $1.5^{\circ} \mathrm{C}$ y $5.5^{\circ} \mathrm{C}$, según de los niveles de emisión de gases de efecto invernadero (World Bank 2014). Adicionalmente, la evidencia existente sugiere que el cambio climático puede estar afectando a la región andina de manera más rápida y pronunciada que en otras regiones. Por ejemplo, se ha reportado que el incremento de temperatura en la región central de los Andes durante las últimas décadas ha sido $70 \%$ mayor que el promedio global (EuropeAid 2009). El incremento de temperatura causará que los meses de calor extremo en cada verano se vuelvan mucho más frecuentes, especialmente a lo largo de las costas de Perú, Ecuador y Colombia (World Bank 2014).

En adición a los patrones de temperatura, los patrones de precipitación se verán también fuertemente afectados en varias regiones de Sudamérica. Países en las costas oeste del continente, entre los cuales se incluyen Ecuador, Colombia y Perú, podrían ver un incremento en precipitación anual de hasta un 30\% hasta el año 2100 (World Bank 2014). Adicionalmente, se prevé un incremento en la frecuencia de eventos de El Niño extremos en la región (Cai et al. 2014).

Efecto del cambio climático en las poblaciones de Aedes aegypti.- El efecto del cambio climático ha sido evaluado en diferentes aspectos de la biología de A. aegypti. Precipitación y temperatura han sido reportados como factores que influencian no solo la dinámica poblacional de esta especie (Barrera et al. 2011, Chowell et al. 2011), sino también su habilidad para transmitir virosis (Watts et al. 1987). Debido a esto, varios estudios se han enfocado en el efecto que las variables ambientales tienen sobre los patrones de distribución geográfica de esta especie. Khormi \& Kumar (2014) elaboraron un análisis predictivo del impacto de cambios de temperatura, humedad, sequía y calor como potenciales efectos del calentamiento global en la distribución de A. aegypti, para finales del siglo XXI. A base de los resultados obtenidos, estos autores prevén una ampliación del rango latitudinal de dispersión de esta especie, lo que permitiría que estos insectos transmisores de enfermedad se diseminen hacia regiones en las que previamente no se encontraban, desde las zonas tropicales a zonas templadas (Khormi \& Kumar 2014).
Adicionalmente, otros estudios han observado cambios en la distribución altitudinal de A. aegypti por efecto del cambio climático. Por ejemplo, en Colombia se consideraba que $A$. aegypti solo habitaba hasta alturas no superiores a los 1500 metros sobre el nivel del mar (msnm). Sin embargo, en el año 1981 se encontraron poblaciones de A. aegypti en 22 localidades por encima de los 1600 msnm, con dos de estas localidades alcanzando los 2 200 msnm (Suárez \& Nelson 1981). Como es de esperarse, la ampliación del rango de distribución de $A$. aegypti hacia nuevas regiones geográficas (ya sea a nivel latitudinal o altitudinal) tiene el potencial de aumentar en esas áreas la incidencia de las enfermedades arbovirales transmitidas por este mosquito (Hopp \& Foley 2001, Hales et al. 2002).

Además de evaluar el efecto del cambio climático en la distribución de A. aegypti, es de extrema importancia evaluar también cómo estos cambios pueden influir en aspectos tales como la fisiología, ecología y comportamiento de esta especie. En este sentido, podemos hacer referencia a varios trabajos que han evaluado los efectos que produce el incremento de la temperatura ambiental en la resistencia a insecticidas en A. aegypti: Polson y colaboradores (2012) evaluaron el efecto de variaciones en la temperatura de crianza de larvas de A. aegypti en la resistencia a insecticidas organofosforados, y determinaron que poblaciones criadas a temperaturas más altas mostraron mayor susceptibilidad a estos químicos.

De la misma manera, se han hecho estudios predictivos de cómo afectarían las variaciones de temperatura a la capacidad vectorial de A. aegypti. La capacidad vectorial describe la habilidad de un vector para propagar enfermedades entre animales vertebrados, tomando en cuenta al hospedero, el agente etiológico, el vector y sus interacciones (Marquardt 2005). Para determinar la capacidad vectorial de una especie se toman en cuenta cinco aspectos: 1) la tasa de picadura diaria del vector, 2) la probabilidad de transmisión de la enfermedad del vector al humano, en cada picadura, 3) la probabilidad que el vector se infecte, en cada picadura, 4) el período de incubación extrínseca del patógeno (duración del período entre el momento de ingestión del patógeno y el momento en el cual un mosquito puede transmitir la enfermedad) y 5) la tasa de mortalidad del vector (Anderson \& Rico-Hesse 2006). Liu-Helmersson y colaboradores (2014) elaboraron un estudio para determinar el efecto de diferentes temperaturas y de la variación de estas temperaturas durante el día (variable denominada "rango de temperatura diurna", o DTR, por sus siglas en inglés), en la capacidad vectorial de A. aegypti. Los autores proponen un modelo no-lineal, en el que las temperaturas extremas -sean estas muy altas o muy bajas- tienden a reducir la capacidad vectorial de A. aegypti. 
Además, determinaron que la temperatura óptima de transmisión es aproximadamente $29^{\circ} \mathrm{C}$. Cuando la temperatura ambiental promedio se aleja de esta temperatura óptima, el efecto de un DTR amplio incrementa la capacidad vectorial de A. aegypti. Además, cuando la temperatura promedio es cercana a la temperatura óptima de transmisión, el efecto de un DTR corto incrementa la capacidad vectorial mientras que un DTR amplio disminuye la capacidad de transmisión de dengue.

Estos resultados están en concordancia con reportes anteriores, los cuales demostraron que la interacción entre las fluctuaciones de temperatura y la capacidad vectorial en A. aegypti es compleja, y depende en gran medida no solamente de la temperatura promedio, sino del grado de variación diaria de temperatura (Lambrechts et al. 2011). Cabe resaltar que interacciones no-lineales entre las fluctuaciones en temperatura y la capacidad vectorial han sido reportados para otras interacciones mosquito/ parásito. Por ejemplo, Mordecai et al. (2012) proponen que en el caso de la malaria (enfermedad transmitida por mosquitos del género Anopheles) la temperatura óptima de transmisión es de $25^{\circ} \mathrm{C}$ y la transmisión declina significativamente una vez que la temperatura ambiental sube por encima de $\operatorname{los} 28^{\circ} \mathrm{C}$.

Basándonos en estos estudios, podemos establecer que a pesar de que el potencial epidémico de las enfermedades transmitidas por A. aegypti es significativamente dependiente de variaciones en la temperatura ambiental, la naturaleza de esta interacción no es lineal, sino extremadamente compleja. Nuestro entendimiento de este crucial aspecto de la epidemiología de las enfermedades arbovirales requiere del desarrollo de nuevos modelos, suficientemente sofisticados como para incorporar datos tanto teóricos como empíricos. Por lo tanto, el desafío actual consiste en identificar qué otros aspectos de la biología y ecología de A. aegypti se verán afectados con el cambio climático, con el fin de proveer información que nos permita modelar los potenciales cambios futuros en la distribución y capacidad vectorial de esta especie, ayudándonos así a enfrentar los retos que encontrarán las campañas de control vectorial.

De lo anteriormente expuesto se desprende que para comprender cómo los futuros cambios en patrones climáticos darán forma al paisaje epidemiológico de las enfermedades transmitidas por A. aegypti es necesario: (a) conocer cuáles son los aspectos biológicos relevantes de esta especie que varían en función del clima, y (b) tener una idea de la dirección y magnitud de los cambios ambientales que pueden esperarse a futuro. A pesar que nuestro conocimiento actual en ambos campos es limitado, la información disponible nos permite visualizar escenarios definitivamente informativos.
Debido a las complejidades inherentes a los modelos predictivos de cambio climático a nivel global, en el presente artículo limitamos nuestra discusión a la región latinoamericana, con especial énfasis en el Ecuador y sus países vecinos. En ese contexto, las predicciones de cambio climático global indican que los países de la costa noroeste de Sudamérica (Colombia, Ecuador y Perú) experimentarán hasta el año 2100 un incremento de hasta un $30 \%$ en su precipitación anual promedio (World Bank 2014). Adicionalmente, durante el mismo período se prevé un incremento en la frecuencia e intensidad de eventos con precipitación extrema, como por ejemplo fenómenos de El Niño inusualmente intensos (Cai et al. 2014, Power et al. 2013). También, se proyecta que no solamente la temperatura promedio del área incrementará de manera significativa (entre $1.5^{\circ} \mathrm{C}$ y $5.5^{\circ} \mathrm{C}$ para el año 2100), sino que períodos de calor extremo se volverán mucho más frecuentes que en la actualidad (World Bank 2014).

Al considerar que la temperatura y la precipitación son los dos aspectos ambientales con la mayor influencia sobre la biología y distribución de A. aegypti, es posible predecir que los cambios climáticos previstos para la región tendrán un impacto significativo en las poblaciones de esta especie de vector. La naturaleza y magnitud de dicho impacto son, sin embargo, difíciles de predecir con exactitud pues responden a una intrincada red de factores. El incremento en la precipitación promedio probablemente se traducirá en una expansión significativa del tamaño poblacional de este insecto en las áreas aptas para su desarrollo. Adicionalmente, no podemos dejar de lado el efecto que el propuesto incremento en la frecuencia de eventos de precipitación extrema puede tener. Eventos de esta naturaleza generalmente causan inundaciones, y producen el deterioro de estructuras tanto públicas como privadas, como por ejemplo caminos, viviendas, escuelas y centros de salud. Esto a su vez se traduce en un incremento de sitios de reproducción para mosquitos, el desplazamiento y hacinamiento de personas afectadas, $\mathrm{y}$ dificultades de acceso a servicios de salud. (todos factores que complican el panorama epidemiológico y favorecen la transmisión de enfermedades, incluyendo aquellas para las que A. aegypti sirve de vector principal).

Predecir los efectos del incremento de temperatura previsto para la zona puede ser un reto mucho más grande. Como hemos expuesto anteriormente, la relación entre temperatura y capacidad vectorial presenta patrones complejos y no necesariamente lineales. En términos generales, podemos asumir que en áreas en donde la temperatura promedio actual se encuentra por debajo de la temperatura que optimiza la capacidad vectorial de A. aegypti, un incremento de temperatura podría significar un incremento en la intensidad de transmisión de 
arbovirosis. Sin embargo, en áreas donde los patrones de temperatura actuales optimizan la capacidad vectorial de A. aegypti (o en lugares que al momento ya presentan temperaturas más altas que las óptimas), el calentamiento provisto para el futuro podría resultar en un decrecimiento de la intensidad de transmisión.

En nuestro país, debido a la complejidad geográfica, meteorológica y social del territorio nacional, el efecto del cambio climático en la transmisión de enfermedades por A. aegypti podría variar ampliamente entre una zona y otra. Predecir la naturaleza y magnitud de estos efectos requerirá el desarrollo de modelos complejos, que incorporen no solamente datos teóricos sino también información empírica y con una resolución espacial adaptada a nuestra geografía. El desarrollo y aplicación de dichos modelos deben convertirse en una prioridad para los investigadores y autoridades de salud, tanto a nivel nacional como regional.

Para terminar, creemos que es necesario recalcar que en adición a $A$. aegypti y sus arbovirus asociados, existen algunas otras especies tanto de insectos vectores como de patógenos cuya biología es también susceptible a las variaciones ambientales. Estos organismos tienen el potencial de convertirse en enemigos importantes de la salud pública a nivel regional y mundial si es que no son monitoreados y controlados adecuadamente.

\section{REFERENCIAS BIBLIOGRÁFICAS}

Allison I, Bindoff NL, Bindschadler RA, Cox PM, de Noblet N, England MH, et al. 2009. The Copenhagen Diagnosis, 2009: Updating the World on the Latest Climate Science. The University of New South Wales Climate Change Research Centre (CCRC), Sydney, Australia, 60pp.

Anderson JR and Rico-Hesse R. 2006. Aedes aegypti vectorial capacity is determined by the infecting genotype of dengue virus. American Journal of Tropical Medicine and Hygiene 75(5): 886-92.

Barrera R, Amador M and MacKay A. 2011. Population Dynamics of Aedes aegypti and Dengue as Influenced by Weather and Human Behavior in San Juan, Puerto Rico. PLoS Neglected Tropical Diseases 5: e1378.

Bar-Zeev M. 1958. The Effect of Temperature on the Growth Rate and Survival of the Immature Stages of Aëdes Aegypti (L.). Bulletin of Entomological Research 49: 157-163.
Bhatt S, Gething P, Brady O, Messina J, Farlow A, Moyes C, Drake J, Brownstein J, Hoen A, Sankoh O, Myers M, George D, Jaenisch T, Wint W, Simmons ., Scott T, Farrar J, Hay S. 2013. The global distribution and burden of Dengue. $\mathrm{Na}$ ture 496: 504-507.

Cai W, Borlace S, Lengaigne M, van Rensch P, Collins M, Vecchi G, Timmermann A, Santoso A, McPhaden MJ, Wu L, England MH, Wang G, Guilyardi E, and Jin F-F. 2014. Increasing Frequency of Extreme El Niño Events due to Greenhouse Warming. Nature Climate Change 5(1): $1-6$.

CDC (Center for Disease Control and Prevention). 2016. Zika virus. Página de Internet: http://www.cdc. gov/zika/index.html. Consultada el 20 de Enero del 2016.

Chowell G, Cazelles B, Broutin H, Munayco C. 2011. The influence of geographic and climate factors on the timing of dengue epidemics in Perú, 1994-2008. BMC Infectious Diseases 11: 164.

Cook G, Zumla A. 2009. Manson's Tropical Diseases. 22ava Edición. Saunders. Londres, Inglaterra. $1830 \mathrm{pp}$.

Duffy M, Chen TH, Hancock W, Powers A, Kool J, Lanciotti R. et al. 2009. Zika Virus Outbreak on Yap Island, Federated States of Micronesia. New England Journal of Medicine 360: 253643.

Epstein P. 2005. Climate change and human health. The New England Journal of Medicine 353: 14331436.

EuropeAid. 2009. Climate Change in Latin America. The European Comission / AGRIFOR Consultores. Bruselas, Bélgica. 118 pp.

Gleick P. 1989. Climate change, hydrology, and water resources. Reviews of Geophysics 27 (3): 329 -344 .

Gould EA, Higgs S. 2009. Impact of climate change and other factors on emerging arbovirus diseases. Transactions of the Royal Society of Tropical Medicine and Hygene 103(2): 109-121.

Gubler D. 2001. Human Arbovirus Infections Worldwide. Annals of the New York Academy of Sciences 951: 13-24 
Hales S, de Wet N, Maindonald $\mathrm{J}$ and Woodward A. 2002. Potential effect of population and climate changes on global distribution of dengue fever: an empirical model. Lancet 360: 830-834.

Hopp M, Foley J. 2001. Global-scale relationships between climate and the dengue fever vector, Aedes aegypti. Climatic Change 48: 441-463.

IPCC (Intergovernmental Panel on Climate Change). 2013. Summary for Policymakers. En: Stocker, T.F., D. Qin, G.-K. Plattner, M. Tignor, S.K. Allen, J. Boschung, A. Nauels, et al. (eds.). Climate Change 2014: The Physical Science Basis. Contribution of Working Group I to the Fifth Assessment Report of the Intergovernmental Panel on Climate Change: 3-29. Cambridge University Press, Cambridge, Inglaterra, y New York, NY, EEUU.

Khormi HM and Kumar L. 2014. Climate change and the potential global distribution of Aedes aegypti: spatial modelling using geographical information system and CLIMEX. Geospatial Health 8(2): 405-415.

Kuhn R, Zhang W, Rossmann M, Pletnev S, Corver J, Lenches E. et al. 2002. Structure of Dengue Virus: Implications for Flavivirus Organization, Maturation, and Fusion. Cell 108: 717725 .

Lambrechts L, Paaijmans KP, Fansiri T, Carrington LB, Kramer LD, Thomas MB and Scott TW. 2011. Impact of daily temperature fluctuations on dengue virus transmission by Aedes aegypti. Proceedings of the National Academy of Sciences of the USA 108(18): 7460-7465.

Liu-Helmersson J, Stenlund H, Wilder-Smith A and Rocklöv J. 2014. Vectorial Capacity of Aedes aegypti: Effects of Temperature and Implications for Global Dengue Epidemic Potential. PlosOne 9(3): 1-10.

Marquardt W. 2005. Biology of Disease Vectors. 2da Edición. Elsevier. EEUU. 785 pp.

Mordecai EA, Paaijmans KP, Johnson LR, Balzer C, Ben-Horin T, de Moor E, McNally A, Pawar S, Ryan SJ, Smith TC and Lafferty KD. 2013. Optimal temperature for malaria transmission is dramatically lower than previously predicted. Ecology Letters 16: 22-30.
MSP (Ministerio de Salud Pública del Ecuador). 2013. Boletín epidemiológico No. 39 de la situación de Dengue en el Ecuador 2013. Página de Internet: http://www.salud.gob.ec/boletin-epidemiologico-no-39-de-la-situacion-de-dengue-en-el-ecuador-2013/. Consultada el 2 de Octubre del 2016.

MSP (Ministerio de Salud Pública del Ecuador). 2015. Gaceta-Dengue-y-Chikungunya-SE-51 2015. Página de Internet: http://www.salud.gob.ec/ wp-content/uploads/2014/09/Graceta-Dengue-y-Chikungunya-SE-51.pdf. Consultada el 2 de Octubre del 2016.

MSP (Ministerio de Salud Pública del Ecuador). 2016a. Gaceta-Dengue-y-Chikungunya-SE-1 2016. Página de Internet: http://www.salud.gob.ec/ wp-content/uploads/2014/09/gaceta-unida-semana-1-_2016.pdf. Consultada el 2 de Octubre del 2016.

MSP (Ministerio de Salud Pública del Ecuador). 2016 b. Alerta epidemiológica: ante la posibilidad de introducción del virus Zika en Ecuador. Página de Internet: http://www.salud.gob.ec/ boletin-de-prensa-alerta-epidemiologica-ante-la-posibilidad-de-introduccion-del-virus-zika-en-ecuador/. Consultada el 2 de Octubre del 2016.

MSP (Ministerio de Salud Pública del Ecuador). 2016c. Subsecretaria de Vigilancia de la Salud Pública. Dirección Nacional de Vigilancia Epidemiológica. Enfermedades Transmitidas Por Vectores. Zika. Ecuador, SE 52 - 53, 2015. SE 1- 6, 2016. Página de Internet: http://www.salud.gob.ec/wp-content/uploads/2015/12/GACETA-ZIKA-SEM-6_viernes.pdf. Consultada el 2 de Octubre del 2016.

MSP (Ministerio de Salud Pública del Ecuador). 2016d. Gaceta Epidemiológica de Enfermedades Transmitidas por Vectores: Zika Virus SE 52-53, 2015. SE 1-33, 2016. Página de Internet: http://www.salud.gob.ec/wp-content/ uploads/2015/12/GACETA-ZIKA-SEM331. pdf. Consultada el 2 de Octubre del 2016.

MSP (Ministerio de Salud Pública del Ecuador). 2016e. Subsecretaria de Vigilancia de la Salud Pública. Dirección Nacional de Vigilancia Epidemiológica. Enfermedades Transmitidas Por Vectores. Fiebre Chikungunya. Semana Epidemiológica 38/2016. Página de Internet: http://www.salud. 
gob.ec/wp-content/uploads/2014/09/GACETA-Chikungunya-38-2016.pdf . Consultada el 2 de Octubre del 2016.

Morin C, Comrie A and Ernst K. 2013. Climate and Dengue Transmission: Evidence and Implications. Environmental Health Perspectives 121 (1112): $1264-1272$.

National Aeronautics and Space Administration (NASA). 2015. Global Climate Change, Vital Signs of the Planet. Página de Internet: http:// climate.nasa.gov.

Ocampo CB, Salazar-Terreros MJ, Mina NJ, McAllister $\mathrm{J}$ and Brogdon W. 2011. Insecticide resistance status of Aedes aegypti in 10 localities in Colombia. Acta Tropica 118(1): 37-44.

OMS (Organización Mundial de la Salud). 2015. Chikungunya. Página de Internet: http://www.who. int/mediacentre/factsheets/fs327/es/. Consultada el 2 de Octubre del 2016.

PAHO (The Pan-American Health Organization. 2016. Number of Reported Cases of Dengue and Severe Dengue (SD) in the Americas, by Country. Página de Internet: http://www.paho.org/ hq/index.php?option=com_docman\&task=doc_view\&Itemid=270\&gid=36293\&lang=en. Consultada el 2 de Octubre del 2016.

Polson K, Brogdon W, Rawlins S. and Chadee D. 2012. Impact of environmental temperatures on resistance to organophosphate insecticides in Aedes aegypti from Trinidad. Revista Panamericana de Salud Pública 32(1): 1-8.

Powers A and Logue C. 2007. Changing patterns of chikungunya virus: re-emergence of a zoonotic arbovirus. Journal of General Virology 88: 2363-2377.

Power S, Delage F, Chung C, Kociuba G and Keay K. 2013. Robust Twenty-first-century Projections of El Niño and Related Precipitation Variability. Nature 502 (7472): 541-45.

Ranson H, Burhani J, Lumjuan N and Black IV W. 2010. Insecticide resistance in dengue vectors. TropIKA 1(1):1-12.

Santer BD, Taylor KE, Wigley TM, Johns TC, Jones PD, Karoly DJ, et al. 1996. A search for human influences on the thermal structure of the atmosphere. Nature 382: 39 - 46.
Suarez MF and Nelson MJ, 1981. Registro de altitud de Aedes aegypti en Colombia. Biomedica 1(4): 225 .

Tun-Lin W, Burkot TR and Kay B. 2000. Effects of temperature and larval diet on development rates and survival of the dengue vector Aedes aegypti in north Queensland, Australia. Medical and Veterinary Entomology 14: 31-37.

Uyar Y. 2013. Arboviral Infections around the World. Journal of Tropical Diseases \& Public Health 1: e105.

Vaughn D, Green S, Kalayanarooj S, Innis B, Nimmannitya S, Suntayakorn S, et al. 2000. Dengue Viremia Titer, Antibody Response Pattern, and Virus Serotype Correlate with Disease Severity. The Journal of Infectious Diseases 181: 2-9.

Watts DM, Burke, D, Harrison, BA, Whitmire RE, Nisalak A. 1986. Effect of Temperature on the Vector Efficiency of Aedes aegypti for Dengue 2 Virus. American Journal of Tropical Medicine and Hygiene 36: 143-152.

WHO (World Health Organization) / TDR (the Special Programme for Research and Training in Tropical Diseases). 2009. Dengue Guidelines for diagnosis, treatment, prevention and control. The World Health Organization. Génova, Suiza. 147 pp.

WHO (World Health Organization). 2015. Impact of Dengue. Página de Internet: http://www.who. int/csr/disease/dengue/impact/en/. Consultada el 16 de Diciembre del 2015.

World Bank. 2014. Turn Down the Heat: Confronting the New Climate Normal. The World Bank Group. Washington DC, EEUU. 275 pp. 